\title{
SAR Target Recognition via Incremental Nonnegative Matrix Factorization
}

\author{
Sihang Dang (iD), Zongyong Cui, Zongjie Cao* (iD) and Nengyuan Liu \\ Center for Information Geoscience, University of Electronic Science and Technology of China, \\ Chengdu 611731, China; dangsihang@163.com (S.D.); zycui@uestc.edu.cn (Z.C.); \\ nengyuanliu@outlook.com (N.L.) \\ * Correspondence: zjcao@uestc.edu.cn
}

Received: 28 December 2017; Accepted: 26 February 2018; Published: 1 March 2018

\begin{abstract}
In synthetic aperture radar (SAR) target recognition, the amount of target data increases continuously, and thus SAR automatic target recognition (ATR) systems are required to provide updated feature models in real time. Most recent SAR feature extraction methods have to use both existing and new samples to retrain a new model every time new data is acquired. However, this repeated calculation of existing samples leads to an increased computing cost. In this paper, a dynamic feature learning method called incremental nonnegative matrix factorization with $L_{p}$ sparse constraints ( $L_{p}$-INMF) is proposed as a solution to that problem. In contrast to conventional nonnegative matrix factorization (NMF) whereby existing and new samples are computed to retrain a new model, incremental NMF (INMF) computes only the new samples to update the trained model incrementally, which can improve the computing efficiency. Considering the sparse characteristics of scattering centers in SAR images, we set the updating process under a generic sparse constraint $\left(L_{p}\right)$ for matrix decomposition of INMF. Thus, $L_{p}$-INMF can extract sparse characteristics in SAR images. Experimental results using Moving and Stationary Target Acquisition and Recognition (MSTAR) benchmark data illustrate that the proposed $L_{p}$-INMF method can not only update models with new samples more efficiently than conventional NMF, but also has a higher recognition rate than NMF and INMF.
\end{abstract}

Keywords: SAR target recognition; incremental learning; incremental NMF; $L_{p}$ sparse constraint

\section{Introduction}

Synthetic aperture radar (SAR) is useful for ground observations, as it can work in the all time and weather conditions. SAR target recognition can use information from images obtained by SAR to determine the category and type of target. SAR target recognition is one of the key technologies that has improved the ability of SAR information sensing and realized the application of SAR technology, especially in military and civil applications. The most recent works in SAR target recognition use images of training samples to obtain a more than 90\% recognition rate without any pretreatment [1-3].

Most of the work on SAR automatic target recognition (ATR) only considers how to use a fixed number of training samples to train a suitable model for recognition tasks. In SAR target recognition, new training samples are obtained continuously, and as such there is a need for the feature model to be updated in real time. However, traditional methods can only train suitable models with a fixed number of samples. As more training samples are gathered, the model has to be retrained with the addition of new data. For example, traditional methods can train a suitable model when there are 100 samples to start with. As the sample number increases, they can also train suitable models when the number of samples is 110,120,130, and so on later. Using both existing and new samples to retrain a new model every time results in a linear increase in computation costs. This leads to reduced data 
processing efficiency . One approach to solving this problem is by developing an incremental learning method to replace the traditional methods.

Incremental learning methods compute data to update the existing training model rather than retraining it [4-6], in a manner similar to human cognition. In recent years, in the fields of video surveillance, facial recognition, and others, many incremental learning methods have been proposed, and can be divided into two aspects based on the process. Firstly, feature extraction methods based on incremental learning can use new data to update the existing feature space efficiently. Incremental principal component analysis (IPCA) is proposed, which is an extension of principal component analysis (PCA) and is applied in the field of facial recognition [7-9]. Incremental linear discriminant analysis (ILDA) is derived, which is based on linear discriminant analysis (LDA) [10]. ILDA optimizes separability of classes incrementally and is applied to facial recognition. Incremental nonnegative matrix factorization (INMF) is proposed to extract subspace representations for each new sample to improve traditional nonnegative matrix factorization (NMF) and is applied to video surveillance tasks [11]. Secondly, a classification method based on incremental learning can use new data to update the decision function, such as incremental support vector machine (ISVM), incremental nearest neighbor classification, incremental neural network, and so on [12-15]. The main advantages of incremental learning are that it saves the storage space of existing samples and improves the speed at which models are updated. However, completely apart from existing samples, incremental learning methods usually lead to a decline in recognition accuracy compared to conventional methods [16].

Since feature extraction is a key process of SAR ATR [17], we aimed to develop a kind of incremental feature extraction method for SAR target features. Compared with other methods of feature extraction, nonnegative matrix factorization (NMF) can obtain local feature representations of the target, which is more in line with the mechanism of human cognition and has better performance than other methods in SAR target recognition $[18,19]$. As mentioned in the introduction, incremental nonnegative matrix factorization (INMF) is an incremental learning method for updating single samples. INMF computes new samples to update the model incrementally, which avoids repetitive learning of existing samples and improves data processing efficiency. However, because it is completely divorced from existing data, the performance of INMF in SAR target recognition is inferior to that of NMF. Thus, we must find a way to improve the accuracy of INMF without increasing computational complexity. Moreover, we need to ensure that the performance of the incremental method is equal to or higher than that of the traditional method.

In addition, due to the scattering center characteristics and target attitude sensitivity of SAR, the basis matrix and coding matrix obtained by NMF should be sparse in SAR [2]. To some extent, NMF with sparse constraints can improve the ability of feature selection and the precision of matrix decomposition $[20,21]$. NMF based on sparse constraints such as $L_{1}$-NMF and $L_{1 / 2}$-NMF can improve the accuracy of SAR target recognition, which has been confirmed by previous work [2]. The authors of [2] propose the novel non-negative matrix factorization (NMF) variant $L_{1 / 2}$-NMF after visualization and analysis of the process of target recognition via NMF for synthetic aperture radar (SAR) images. These authors [2] show that both the basis and coding matrices obtained by $L_{1 / 2}$-NMF have higher sparseness than those obtained by NMF, $L_{1}-N M F$, and NMF with sparseness constraints (NMFsc), and recognition results demonstrate that the $L_{1 / 2}$-NMF outperforms $L_{1}-\mathrm{NMF}$, NMFsc and non-smooth NMF.

Considering sparse characteristics of scattering centers in a SAR image, a new INMF method based on generalized sparse constraints is proposed. This method, called $L_{p}$-INMF, adds $L_{p}$ sparse constraints to the decomposition matrix during the update process, obtaining more accurate solutions than general INMF. The $p$ is typically between 0 and 1 so that different norm sparse constraints can be used. Experimental results on Moving and Stationary Target Acquisition and Recognition (MSTAR) benchmark data illustrate that $L_{p}$-INMF can not only compute gradually increasing numbers of samples more efficiently than traditional non-incremental NMF and incremental PCA (IPCA), but can also obtain a higher recognition rate than NMF and incremental NMF (INMF). 
The rest of the paper is organized as follows. In Section 2, related works are introduced. Section 3 summarizes the proposed $L_{p}$-INMF and the whole SAR target incremental recognition process based on $L_{p}$-INMF. Section 4 shows the experimental results of the proposed method based on the MSTAR data set. Section 5 concludes this paper.

\section{Related Works}

\subsection{Nonnegative Matrix Factorization}

This section is used to introduce the formula and symbol representation of NMF.

We assume that a SAR target sample is represented by an $m$ dimensional vector and the number of training samples is equal to $n$. The data matrix, $V$, will be an $\mathrm{m}$ by $n$ matrix where $V_{i j}$ refers to an entity in $V(i=1, \ldots, m, j=1, \ldots, n)$. NMF approximately factors the data matrix $\left(V \in R^{m \times n}\right)$ into two matrices: the base data, $W \in R^{m \times r}$, and the encoding matrix, $H \in R^{r \times n}$. Each column vector in $V$ has a corresponding representation in the coding matrix. The $r$ is a pre-defined parameter which is designed as the rank of the factorization and determines the level of dimension reduction.

Conventionally the NMF method minimizes a cost function regarding the reconstruction error defined by Equation (1).

$$
F(W, H)=\frac{1}{2}\|V-W H\|^{2}
$$

This error function $F$ is a convex function of $W$ and $H$ separately. Therefore, multiplicative and alternating update rules for $W$ and $H$ are derived via gradient descent optimization. The update rules for the elements of matrix $W$ and $H$ are given by Equations (2) and (3), where $\mu=1, \ldots, r$.

$$
\begin{aligned}
& W_{i, \mu} \leftarrow W_{i, \mu} \frac{\left(V H^{T}\right)_{i, \mu}}{\left(W H H^{T}\right)_{i, \mu}} \\
& H_{\mu, j} \leftarrow H_{\mu, j} \frac{\left(W^{T} V\right)_{\mu, j}}{\left(W^{T} W H\right)_{\mu, j}}
\end{aligned}
$$

Since each column of data matrix $V$ corresponds to a different sample, the arrival of each new sample leads to an additional column in $V$. It is well known that the computational complexity of a standard NMF is $\mathrm{O}(\mathrm{mnr})$ per iteration, which implies that the computational cost increases linearly with the number of columns of data. Therefore, whenever a new sample is received, re-running the existing and new samples is not an approach which meets the demands of model updating.

\subsection{Incremental NMF for SAR Target Recognition}

In this subsection, INMF is introduced to overcome the efficiency problem of NMF for updating the model.

The idea of incremental nonnegative matrix factorization is to update the decomposition matrices $W$ and $H$ with the addition of a new sample without increasing the computational cost. In this process, a column representing a new sample has been added to the sample matrix $V$ and the coding matrix $H$, and then the matrix $W$ is updated with the new sample [11].

The optimized factor matrices of the existing $k$ samples are denoted by $W_{k}$ and $H_{k}$. In Equation (4), $F_{k}$ refers to the cost function corresponding to NMF representation of the existing $k$ samples.

$$
F_{k}=\left\|V-W_{k} H_{k}\right\|^{2}=\sum_{i=1}^{m} \sum_{j=1}^{k}\left(V_{i, j}-\left(W_{k} H_{k}\right)_{i, j}\right)^{2}
$$


When the $(k+1)$ th sample, $v_{k+1}$, is added, the reconstruction error is formulated by Equations (5) and (6).

$$
\begin{gathered}
F_{k+1}=\left\|V-W_{k+1} H_{k+1}\right\|^{2} \\
=\sum_{i=1}^{m} \sum_{j=1}^{k+1}\left(V_{i, j}-\left(W_{k+1} H_{k+1}\right)_{i, j}\right)^{2} \\
=\sum_{i=1}^{m} \sum_{j=1}^{k}\left(V_{i, j}-\left(W_{k+1} H_{k+1}\right)_{i, j}\right)^{2} \\
+\sum_{i=1}^{m}\left(\left(v_{k+1}\right)_{i}-\left(W_{k+1} h_{k+1}\right)_{i}\right)^{2} \\
\cong F_{k}+f_{k+1} \\
f_{k+1}=\frac{1}{2} \sum_{i=1}^{m}\left(\left(v_{k+1}\right)_{i}-\left(W_{k+1} h_{k+1}\right)_{i}\right)^{2}
\end{gathered}
$$

Thus, when the $(k+1)$ th sample, $v_{k+1}$, is added, the cost function of INMF can be divided into $F_{k}$ and $f_{k+1}$ approximately. $f_{k+1}$ is the part which is relevant to the $(k+1)$ th sample. Thus, when the new sample is added, the existing model can be updated with the new information, instead of being retrained. Let $\frac{\partial F_{k+1}}{\partial\left(W_{k+1}\right)_{i \mu}}=0, \frac{\partial F_{k+1}}{\partial\left(h_{k+1}\right)_{\mu}}=0$. The iterative rules of matrix $W_{k+1}$ and column $h_{k+1}$ are derived as follows:

$$
\begin{gathered}
\left(W_{k+1}\right)_{i \mu} \leftarrow\left(W_{k+1}\right)_{i \mu} \frac{\left(V_{k} H_{k}^{T}+v_{k+1} h_{k+1}^{T}\right)_{i \mu}}{\left(W_{k+1} H_{k} H_{k}^{T}+W_{k+1} h_{k+1} h_{k+1}^{T}\right)_{i \mu}} \\
\left(h_{k+1}\right)_{\mu} \leftarrow\left(h_{k+1}\right)_{\mu} \frac{\left(W_{k+1}^{T} v_{k+1}\right)_{\mu}}{\left(W_{k+1}^{T} W_{k+1} h_{k+1}\right)_{\mu}}
\end{gathered}
$$

The optimization equations of NMF and INMF computing the new samples are shown in Figure 1. The computational complexity is also shown in the Table 1 . The training of existing $\mathrm{k}$ samples has been completed. Figure 1 describes the processes of INMF updating and NMF retraining when the $(k+1)$ th sample comes. Blue represents the existing part and yellow part represents the updating part. When the number of training samples increases, conventional NMF must compute all existing and new samples to retrain, but INMF just computes the new sample to update the model incrementally. Therefore, INMF can avoid repetitive learning existing training samples and improve data processing efficiency.

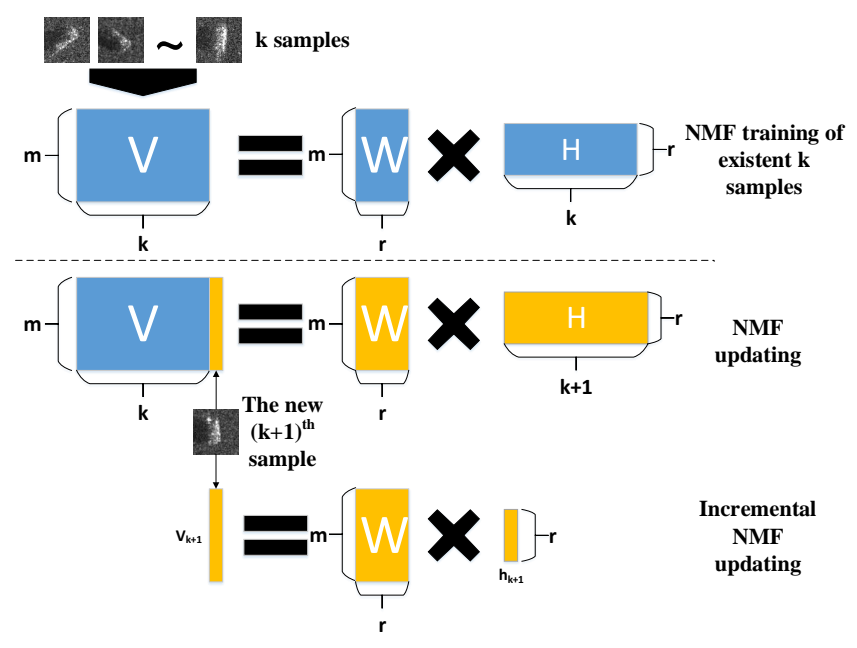

Figure 1. The $(k+1)$ th sample updating process comparison of traditional nonnegative matrix factorization (NMF) and incremental NMF (INMF) when existing k samples have been trained by NMF. 
Table 1. Computational complexity of NMF and INMF for computing the new sample.

\begin{tabular}{cc}
\hline Method & Computational Complexity for Computing the $(k+1)$ th Sample \\
\hline NMF & $\mathrm{O}(\mathrm{m}(\mathrm{k}+1) \mathrm{r})$ \\
INMF & $\mathrm{O}(\mathrm{mr})$ \\
\hline
\end{tabular}

Note that in the cost function of INMF,

$$
F_{k}=\sum_{i=1}^{m} \sum_{j=1}^{k}\left(V_{i, j}-\left(W_{k} H_{k}\right)_{i, j}\right)^{2} \cong \sum_{i=1}^{m} \sum_{j=1}^{k}\left(V_{i, j}-\left(W_{k+1} H_{k+1}\right)_{i, j}\right)^{2}
$$

assuming that the first $k$ columns of $\left(W_{k+1} H_{k+1}\right)$ would be approximately equal to $\left(W_{k} H_{k}\right)$ in Equation (9). Hence, $F_{k+1}$ can be divided into $F_{k}$ and $f_{k+1}$ approximately as mentioned above. The approximation of the cost function of INMF creates an error. The matrix resolution accuracy when updating is lower than that of NMF retraining. While the error cannot be avoided, we can use SAR target sparseness to improve the matrix resolution accuracy.

Our previous study proposed a novel incremental nonnegative matrix factorization (INMF) and experiments were carried out with respect to recognition performance and efficiency in order to overcome the defects that conventional methods have in online processing. For details, please refer to [22].

\section{3. $L_{p}$-INMF Method for SAR Target Recognition}

\subsection{Incremental NMF with $L_{p}$ Sparse Constraint}

Related work [2] has illustrated that the base matrix $W$ and the coding matrix $H$ of NMF should be sparse in SAR. Representing the SAR targets by methods with sparse constraints on the base matrix and coding matrix are beneficial for SAR target recognition [19]. Thus, we derive a new INMF method based on $L_{p}$ constraints for SAR target training model updating. The $p$ is typically between 0 and 1 so that different norm sparse constraints can be used. In the following sections, we analyze the cost function, the update rule and the realization process of the proposed $L_{p}$-INMF.

\subsubsection{Formulating the Cost Function of $\mathrm{L}_{p}$-INMF}

The optimized factor matrices of the existing $k$ samples are denoted by $W_{k}$ and $H_{k}$, where $k \geq 2 r$. In Equation (10), $F_{k}$ refers to the cost function corresponding to the $L_{p}$-NMF representation of the first $k$ samples. $h_{j}$ represents the $j$ th column of $H$, which is relevant to the $j$ th sample.

$$
\begin{aligned}
F_{k} & =\frac{1}{2}\left\|V_{k}-W_{k} H_{k}\right\|^{2}+\lambda \sum_{j=1}^{k}\left\|h_{j}\right\|_{p}^{p} \\
& =\frac{1}{2} \sum_{i=1}^{m} \sum_{j=1}^{k}\left(V_{i, j}-\left(W_{k} H_{k}\right)_{i, j}\right)^{2}+\lambda \sum_{j=1}^{k}\left\|h_{j}\right\|_{p}^{p}
\end{aligned}
$$

where

$$
\left\|h_{j}\right\|_{p}^{p}=\sum_{i=1}^{r} h_{j}(i)^{p}
$$

and $h_{j}(i)$ is the $i$ th element of $h_{j}$. 
When the $(k+1)$ th sample $v_{k+1}$ is added, the reconstruction error is formulated by Equations (12) and (13).

$$
\begin{aligned}
F_{k+1} & =\frac{1}{2}\left\|V_{k+1}-W_{k+1} H_{k+1}\right\|^{2}+\lambda \sum_{j=1}^{k+1}\left\|h_{j}\right\|_{p}^{p} \\
& =\frac{1}{2} \sum_{i=1}^{m} \sum_{j=1}^{k}\left(V_{i, j}-\left(W_{k+1} H_{k+1}\right)_{i, j}\right)^{2}+\lambda \sum_{j=1}^{k}\left\|h_{j}\right\|_{p}^{p} \\
& +\frac{1}{2} \sum_{i=1}^{m}\left(\left(v_{k+1}\right)_{i}-\left(W_{k+1} h_{k+1}\right)_{i}\right)^{2}+\lambda\left\|h_{k+1}\right\|_{p}^{p} \\
& \approx F_{k}+f_{k+1} \\
f_{k+1} & =\frac{1}{2} \sum_{i=1}^{n}\left(\left(v_{k+1}\right)_{i}-\left(W_{k+1} h_{k+1}\right)_{i}\right)^{2}+\lambda\left\|h_{k+1}\right\|_{p}^{p}
\end{aligned}
$$

Thus, when the $(k+1)$ th sample $v_{k+1}$ is added, the $p$ norm constraints of the coding matrix can be divided into two parts. Therefore, the cost function of $L_{p}$-INMF can also be divided into $F_{k}$ and $f_{k+1}$, similar to INMF.

\subsubsection{Inferring the Multiplicative Update Rules of $\mathrm{L}_{p}$-INMF}

After formulating the cost function of $L_{p}-\mathrm{INMF}$, as shown in Equations (14) and (15), a gradient descent method is used to derive the update rule of $L_{p}$-INMF. Whenever a new sample is added, the update variables of the cost function are the base matrix $W$ and the corresponding column $h_{k+1}$ of the new samples in the coding matrix $H$. The calculation procedure is as follows:

$$
\begin{aligned}
& \frac{\partial F_{k+1}}{\partial\left(W_{k+1}\right)_{i \mu}}=\frac{\partial}{\partial\left(W_{k+1}\right)_{i \mu}}\left[\sum_{i=1}^{m} \sum_{j=1}^{k+1}\left(V_{i j}-\left(W_{k+1} H_{k+1}\right)_{i j}\right)^{2}\right] \\
& =\sum_{j=1}^{k+1}\left(-V_{i j}\left(H_{k+1}\right)_{\mu j}+\left(H_{k+1}\right)_{\mu j}\left(W_{k+1} H_{k+1}\right)_{i j}\right) \\
& \frac{\partial F_{k+1}}{\partial\left(h_{k+1}\right)_{\mu}}=\frac{\partial f_{k+1}}{\partial\left(h_{k+1}\right)_{\mu}} \\
& =-\left(W_{k+1}^{T} v_{k+1}\right)_{\mu}+\left(W_{k+1}^{T} W_{k+1} h_{k+1}\right)_{\mu}+\lambda \frac{\partial\left(\left\|h_{k+1}\right\|_{p}^{p}\right)}{\partial\left(h_{k+1}\right)_{\mu}} \\
& =-\left(W_{k+1}^{T} v_{k+1}\right)_{\mu}+\left(W_{k+1}^{T} W_{k+1} h_{k+1}\right)_{\mu} \\
& +\lambda p\left(\left(h_{k+1}\right)_{\mu}\right)^{p-1}
\end{aligned}
$$

Let $\frac{\partial F_{k+1}}{\partial\left(W_{k+1}\right)_{i \mu}}=0, \frac{\partial F_{k+1}}{\partial\left(h_{k+1}\right)_{\mu}}=0$. The iterative rules of matrix $W_{k+1}$ and column $h_{k+1}$ are derived:

$$
\begin{gathered}
\left(W_{k+1}\right)_{i \mu} \leftarrow\left(W_{k+1}\right)_{i \mu} \frac{\left(V_{k} H_{k}^{T}+v_{k+1} h_{k+1}^{T}\right)_{i \mu}}{\left(W_{k+1} H_{k} H_{k}^{T}+W_{k+1} h_{k+1} h_{k+1}^{T}\right)_{i \mu}} \\
\left(h_{k+1}\right)_{\mu} \leftarrow\left(h_{k+1}\right)_{\mu} \frac{\left(W_{k+1}^{T} v_{k+1}\right)_{\mu}}{\left(W_{k+1}^{T} W_{k+1} h_{k+1}\right)_{\mu}+\lambda p\left(\left(h_{k+1}\right)_{\mu}\right)^{p-1}}
\end{gathered}
$$

We complete the addition of $L_{p}$ sparse constraints to the decomposition matrices in the update process by using Equations (16) and (17).

After the end of each new sample update, it is necessary to store the history information for the next update. The storage matrixes are given by Equations (18) and (19).

$$
A=V_{k+1} H_{k+1}^{T}=V_{k} H_{k}^{T}+v_{k+1} h_{k+1}^{T}
$$




$$
B=H_{k+1} H_{k+1}^{T}=H_{k} H_{k}^{T}+h_{k+1} h_{k+1}^{T}
$$

In summary, the updating process of $L_{p}$-INMF is shown in Figure 2. The base matrix $W_{\text {new }}$ and $h_{\text {new }}$ are regularly updated, along with the addition of new samples. The last $W_{\text {new }}$ is the final result of this incremental learning process which can be used in feature extraction.

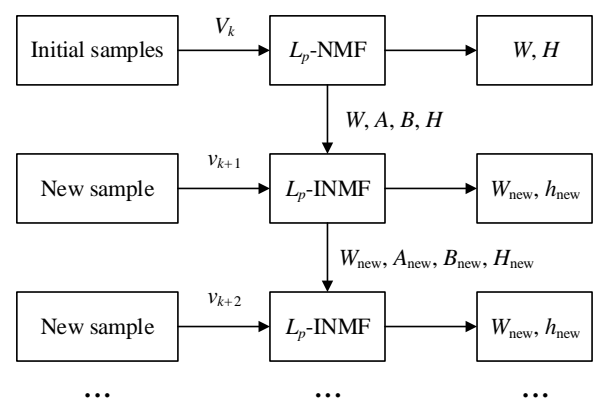

Figure 2. The updating process of $W_{\text {new }}$ and $h_{\text {new }}$ in incremental nonnegative matrix factorization with $L_{p}$ sparse constraints $\left(L_{p}\right.$-INMF).

\subsection{The SAR Target Training Model Updating Process}

The process of updating a SAR target training model is completely different from traditional SAR target recognition. This section describes the incremental learning process of new samples and gives the SAR target training model updating process based on $L_{p}$-INMF.

In the last section, the calculation process based on $L_{p}$-INMF is introduced. $L_{p}-\mathrm{NMF}$ is used to calculate the basic matrix $W$ of the existing samples, and the new base matrix $W_{\text {new }}$ is updated by $L_{p}$-INMF as the number of new samples increases.

As described in the introduction, we use an incremental learning method to compute projection matrix $W$ in a time-consuming feature extraction process. In SAR target recognition based on traditional $\mathrm{NMF}$, the coding matrix $H$ is the feature matrix of training samples. However in INMF, error of $W$ and $H$ accumulates gradually as the number of new samples increased. After incremental learning with a new sample, $h_{k+1}$ could not be used as a feature vector of the new sample. Therefore, when all the new samples have been incorporated and the update is complete, the final base matrix $W_{\text {new }}$ is used to map all the training data in $L_{p}$-INMF. The formula is as follows:

$$
H_{\text {train }}=W_{\text {new }}^{-1} V_{\text {train }}=\left(W_{\text {new }}^{T} W_{\text {new }}\right)^{-1} W_{\text {new }}^{T} V_{\text {train }}
$$

where $V_{\text {train }}$ represents all existing and new samples.

After getting the characteristic $H_{\text {train }}$ of the training sample, it is sent to the support vector machine (SVM) to train the classifier to complete the training process [23,24]. The SVM-based Radial Basis Function (RBF) kernel function is used to classify the feature vector $h_{t e s t}$ of the test sample to obtain the final result. We also use the final base matrix $W_{\text {new }}$ to calculate the feature vector $h_{\text {test }}$ of the test sample $v_{\text {test }}$, as shown in Equation (21).

$$
h_{\text {test }}=W_{\text {new }}^{-1} v_{\text {test }}=\left(W_{\text {new }}^{T} W_{\text {new }}\right)^{-1} W_{\text {new }}^{T} v_{\text {test }}
$$

In summary, the SAR target training model updating process based on $L_{p}$-INMF and traditional NMF are compared in Figure 3. 


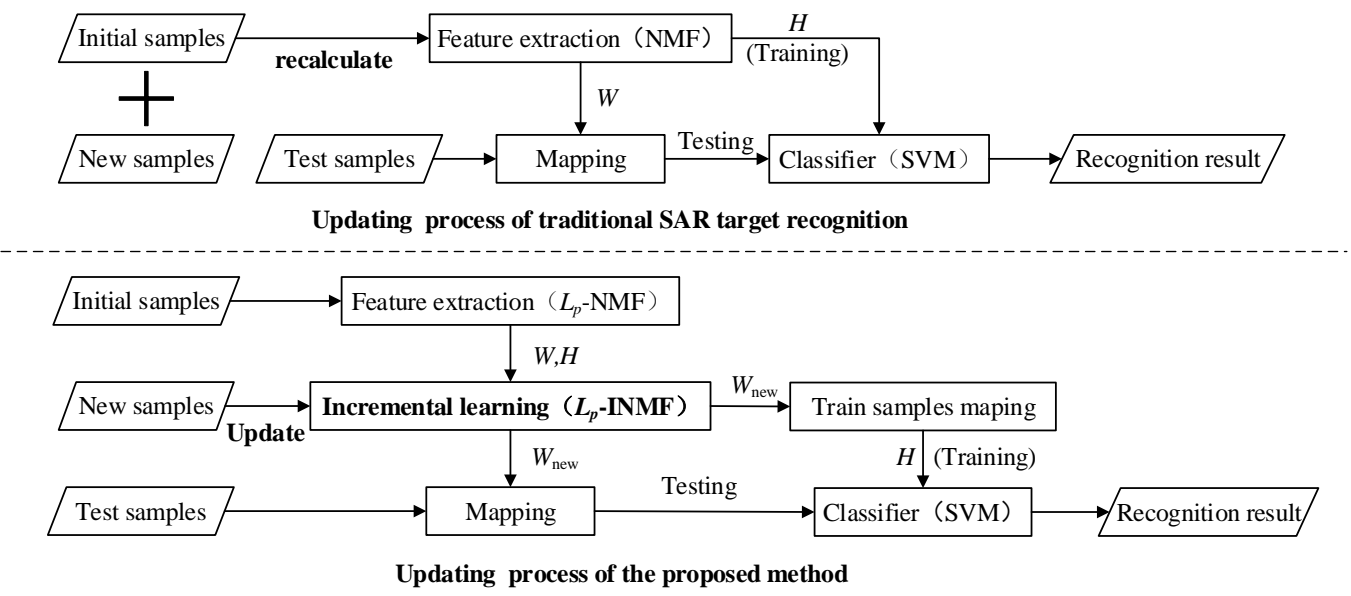

Figure 3. Synthetic aperture radar (SAR) target training model updating process based on traditional NMF and our $L_{p}$-INMF.

As can be seen from the schematic, there are two main improvements with our method. Firstly, the proposed $L_{p}$-INMF uses incremental learning in time-consuming pivotal feature extraction to avoid repetitive computing of existing samples, which improves data processing efficiency. Secondly, the proposed $L_{p}$-INMF adds $L_{p}$ sparse constraints to the decomposition matrices in the update process, which can result in more accurate solutions than traditional INMF and improve the recognition performance.

\section{Experiments}

The SAR images used in our experiments were taken from the MSTAR public database and consist of X-band and $\mathrm{HH}$ polarized SAR images with 0.3-m resolution for multiple targets [25]. These targets include BMP2 (tank), BTR70 (armored car), T72 (tank), BTR60 (armored car), 2 S1 (cannon), BRDM2 (truck), D7 (bulldozer), T62 (tank), ZIL131 (truck), and ZSU23/4 (cannon). The images were captured at two different depression angles $\left(15^{\circ}\right.$ and $\left.17^{\circ}\right)$ with $190 \sim 300$ different aspect versions, which provide full aspect coverage over $360^{\circ}$ [26]. Similar to the earlier experiments, the images with depression angle of $17^{\circ}$ are used as training set, and the images with depression angle of $15^{\circ}$ are used for the test, as shown in Table 2 [27].

Table 2. Summary of the Moving and Stationary Target Acquisition and Recognition (MSTAR) database.

\begin{tabular}{|c|c|c|c|c|c|c|c|c|c|c|c|c|c|c|c|}
\hline Target Class & & 1 & & 2 & & 3 & & 4 & 5 & 6 & 7 & 8 & 9 & 10 & SUM \\
\hline Target Type & sn-9563 & $\begin{array}{l}\text { BMP2 } \\
\text { sn-9566 }\end{array}$ & sn-c21 & $\begin{array}{l}\text { BTR70 } \\
\text { sn-c71 }\end{array}$ & sn-132 & $\begin{array}{c}\text { T72 } \\
\text { sn-812 }\end{array}$ & sn-s7 & BTR60 & $2 \mathrm{~S} 1$ & BRDM2 & D7 & T62 & ZIL131 & ZSU23/4 & \\
\hline Test & 195 & 196 & 196 & 196 & 196 & 195 & 191 & 195 & 274 & 274 & 274 & 273 & 274 & 274 & 3203 \\
\hline
\end{tabular}

We read the image from the RAW format slice data. The original size of the RAW data is large and ranges from 100 to 200. The difference is the size of the scene around the target. All the images in the following experiments are cropped by extracting $64 \times 64$ patches from the center of the image but without any other preprocessing. Optical and SAR images of ten kinds of target samples are shown in Figure 4. 

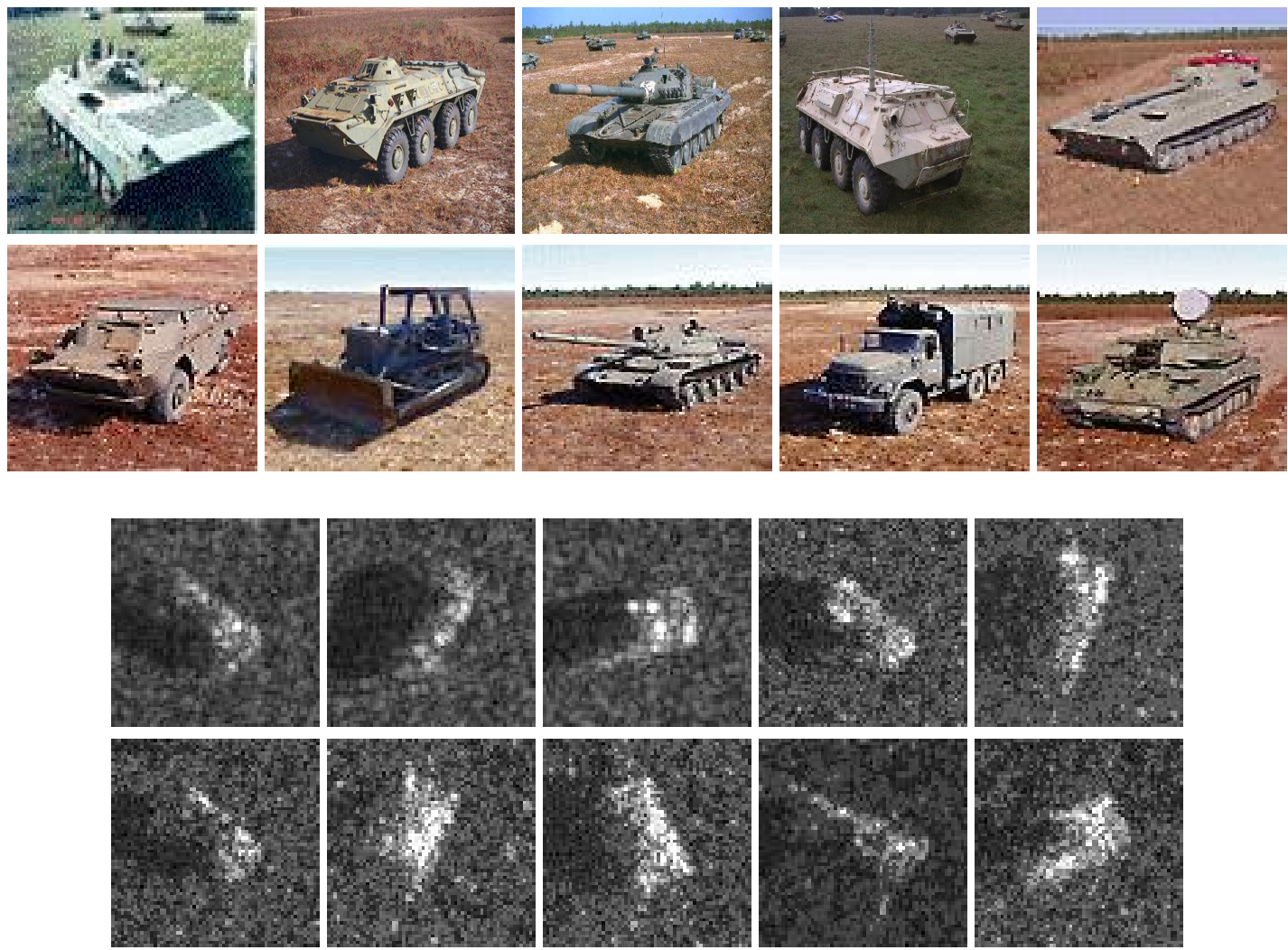

Figure 4. Optical and SAR images of ten kinds of target samples, in the order: BMP2, BTR70, T72, BTR60, 2S1, BRDM2, D7, T62, ZIL131, and ZSU23/4.

\subsection{Experimental Requirements}

To simulate the practical use of the update learning process of the SAR target recognition, specific experimental requirements are set as follows:

(1) The number of existing training samples is 497 , and the remaining training samples are new samples. In practice, new samples are obtained continually in batches; therefore, we establish one batch containing 250 samples. Every time a batch of new training samples is added to update the training model, all the test samples are used to verify the recognition performance and efficiency.

(2) In the contrast experiment, the recognition ability of our $L_{p}$-INMF and traditional NMF is compared when the number of training samples is increased, as described in experimental requirement (1).

(3) Since training samples are randomly obtained in real-life applications, the order of the training samples is randomized multiple times to simulate how samples are obtained in the real life, and in order to conduct several experiments using the same batch of experimental samples..

(4) Non-incremental $L_{1}$-NMF and $L_{1 / 2}$-NMF are successfully applied in SAR target recognition. Thus, the $p$ of $L_{p}$-INMF is set to 1 and $\frac{1}{2}$ in the experiments.

(5) In order to ensure the comparison in experiments is fair, the dimension of feature extraction is always set to 40 .

\subsection{Experiments on Efficiency}

Experiments are repeated ten times and the average training times are recorded. Details of training times of the incremental learning process are shown in Figure 5. The proposed method ( $\left.L_{p}-\mathrm{INMF}\right)$ is compared with NMF. 


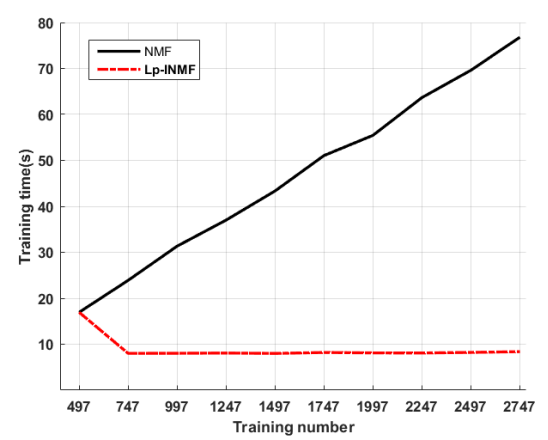

Figure 5. Efficiency comparison of conventional NMF and our $L_{p}$-INMF.

To verify that the proposed $L_{p}$-INMF can improve the accuracy of INMF without increasing computational complexity, the computation times when updating a single sample for IPCA INMF, $L_{1}$-INMF, and $L_{1 / 2}$-INMF are shown in Figure 6 when the number of existing samples is 200.

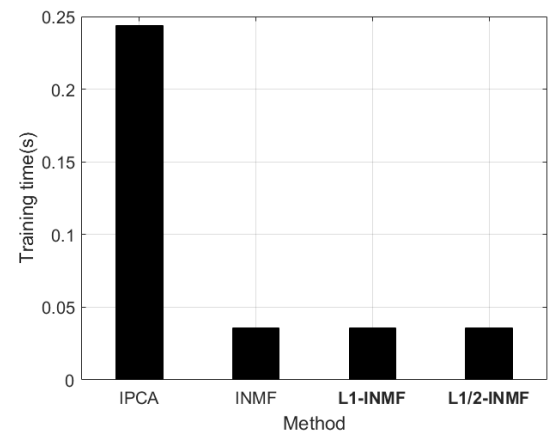

Figure 6. Computation time for updating a single sample under incremental principal component analysis (IPCA), INMF, $L_{1}$-INMF, and $L_{1 / 2}$-INMF.

\subsection{Experiments on the Error of the Decomposition Value}

Previous work [2] has detailed sparseness analysis of base matrices obtained by non-incremental $\mathrm{NMF}, L_{1}-\mathrm{NMF}$, and $L_{1 / 2}$-NMF. Thus, we only measure the precision of the extracted base matrix $W$ and the coding matrix $H$ to evaluate the sparse constraint of INMF as the number of samples continuously increases.

This evaluation method is similar to that found in [20]. Two indexes are used to measure the errors of the extracted base matrix $W$ and the coding matrix $H$, which are represented by the vector angle error (VAE) and root-mean-square error (RMSE), respectively. For the $\mu$ th $(1 \leq \mu \leq r)$ base vector, the true value is $W_{\mu}^{*}$. Then, the VAE between the estimate $W_{\mu}$ and the actual value $W_{\mu}^{*}$ is defined as

$$
V A E_{\mu}=\cos ^{-1}\left(\frac{W_{\mu}^{* T} W_{\mu}}{\left\|W_{\mu}^{*}\right\|\left\|W_{\mu}\right\|}\right)
$$

For the $\mu$ th projection value of the $\mu$ th base vector, the calculated theoretical value is $H_{\mu}^{*}$. The RMSE between the estimate $H_{\mu}$ and the actual value $H_{\mu}^{*}$ is represented by the RMSE, defined as

$$
\operatorname{RMSE}_{\mu}=\left(\frac{1}{N}\left|H_{\mu}^{*}-H_{\mu}\right|^{2}\right)^{1 / 2}
$$

where $N$ is the number of samples.

The definitions in Equations (22) and (23) refer to $W^{*}$ and $H^{*}$, which are ground truth. The ground truth is derived from the direct matrix operation rather than the INMF iteration. For example, 
$W^{*}=V H^{+}$and $H^{*}=W^{+} V . V$ is the data matrix, $W, H$ are the iterative values of INMF, and "+" means pseudo-inverse. The total VAE of the base matrix $W$ and the total RMSE of the coding matrix $H$ are given as follows:

$$
\begin{aligned}
V A E & =\sum_{\mu=1}^{r} V A E_{\mu} \\
R M S E & =\sum_{\mu=1}^{r} R M S E_{\mu}
\end{aligned}
$$

Since the calculated theoretical value is relative, there are two points with respect to index comparison. First, low index values mean better precision of matrix factorization. Second, if the matrix factorization precision is good, the indexes of the two decomposition matrix $W$ and $H$ should be low at the same time.

Two hundred existing samples were used, and 498 new samples were introduced gradually. After computing each sample, the VAE and RMSE were calculated to evaluate the error of the two decomposition matrices $W$ and $H$. Experiments were repeated ten times, and the average values of the two indexes were recorded. The experimental results of $L_{p}-\mathrm{INMF}\left(L_{1}-\mathrm{INMF}\right.$ and $\left.L_{1 / 2}-\mathrm{INMF}\right)$ and traditional INMF are compared in Figures 7 and 8.

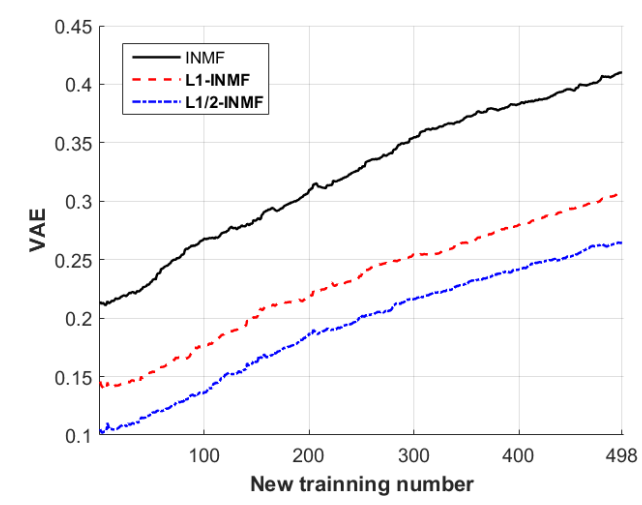

Figure 7. Vector angle error (VAE) comparison of INMF, our $L_{1}$-INMF, and $L_{1 / 2}$-INMF.

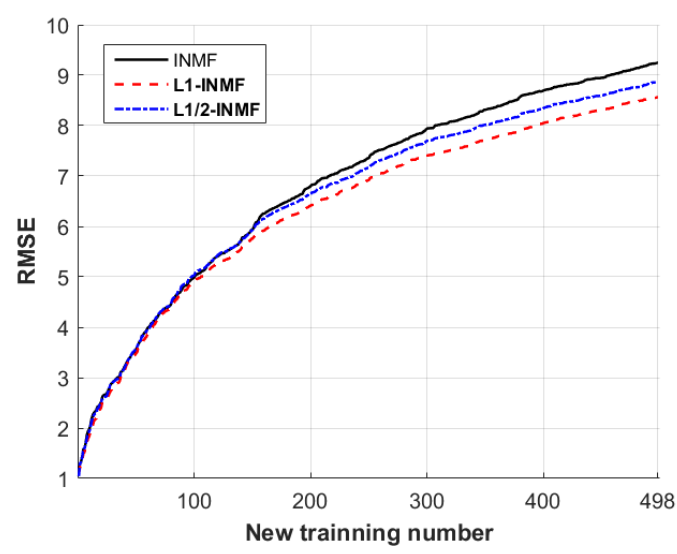

Figure 8. Root-mean-square error (RMSE) comparison of INMF, our $L_{1}$-INMF, and $L_{1 / 2}$-INMF.

\subsection{Experiments on Recognition Performance}

To verify that the sparsity constraint can improve the performance of SAR target recognition, the target recognition accuracies for ten classes using three non-incremental methods (NMF, $L_{1}-\mathrm{NMF}$, and $L_{1 / 2}-\mathrm{NMF}$ ) are shown in Table 3. 
Table 3. Average accuracies of three non-incremental methods (NMF, $L_{1}-\mathrm{NMF}$ [2], and $L_{1 / 2}-\mathrm{NMF}$ [2]), in MSTAR data.

\begin{tabular}{cccc}
\hline Method & NMF & $\boldsymbol{L}_{1}$-NMF [2] & $\boldsymbol{L}_{1 / 2}$-NMF [2] \\
\hline Accuracy & $91.29 \%$ & $92.84 \%$ & $93.41 \%$ \\
\hline
\end{tabular}

In Table 3, it can be seen that NMF with the $L_{p}$ sparse constraint obtains higher accuracy than traditional NMF when $p$ is set to 1 and $\frac{1}{2}$. $L_{1 / 2}$-NMF obtains the best accuracy, which is verified by the previous study [19].

Experiments were repeated ten times, and the average values of recognition rates were recorded as the number of training samples increased. Recognition rates of the incremental learning process are shown in Figure 9. Incremental INMF is compared with non-incremental methods (NMF) in Figure 9. When $p$ was set to 1 and $\frac{1}{2}$, two incremental methods with $L_{p}$ sparse constraint (our $L_{1}$-INMF and $L_{1 / 2}$-INMF) were compared with traditional incremental NMF. The best recognition rates of three incremental methods (INMF, our $L_{1}$-INMF, and $L_{1 / 2}$-INMF) for every class are shown in Table 4.

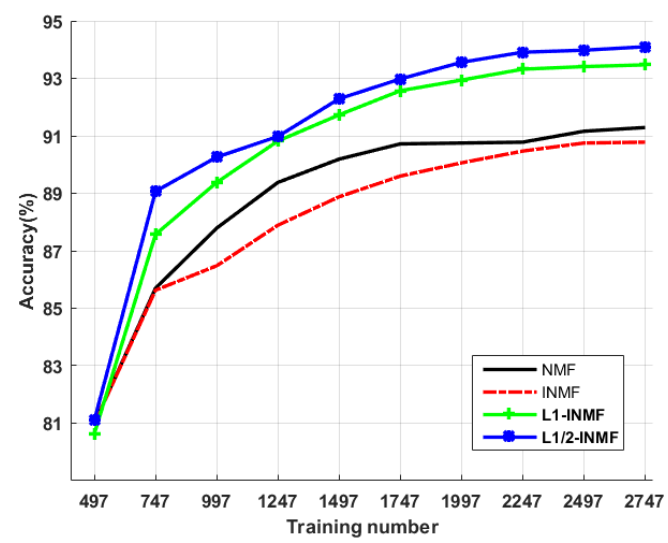

Figure 9. Accuracy comparison of NMF, INMF, $L_{1}-\mathrm{INMF}$, and $L_{1 / 2}-\mathrm{INMF}$, as the number of training samples increased.

Table 4. The recognition performance for ten classes using three incremental methods (INMF, $L_{1}-\mathrm{INMF}$, and $L_{1 / 2}$-INMF).

\begin{tabular}{cccccccccccc}
\hline Method & BMP2 & BTR70 & T72 & BTR60 & 2S1 & BRDM2 & D7 & T62 & ZIL131 & ZSU23/4 & Avg. \\
\hline INMF & $87.56 \%$ & $97.25 \%$ & $87.07 \%$ & $90.84 \%$ & $89.05 \%$ & $88.32 \%$ & $95.98 \%$ & $90.47 \%$ & $95.61 \%$ & $96.71 \%$ & $90.78 \%$ \\
$L_{1}$-INMF & $91.65 \%$ & $98.47 \%$ & $89.00 \%$ & $\mathbf{9 3 . 8 5 \%}$ & $93.07 \%$ & $90.88 \%$ & $97.45 \%$ & $\mathbf{9 5 . 2 4} \%$ & $98.18 \%$ & $98.18 \%$ & $93.69 \%$ \\
$L_{1 / 2}$-INMF & $\mathbf{9 2 . 3 3 \%}$ & $\mathbf{9 8 . 9 8 \%}$ & $\mathbf{8 9 . 8 6 \%}$ & $93.33 \%$ & $\mathbf{9 3 . 4 3 \%}$ & $\mathbf{9 1 . 2 4} \%$ & $\mathbf{9 9 . 2 7} \%$ & $94.87 \%$ & $\mathbf{9 8 . 5 4 \%}$ & $\mathbf{9 9 . 6 4 \%}$ & $\mathbf{9 4 . 3 2} \%$ \\
\hline
\end{tabular}

In order to verify that feature updating led to recognition performance improvement as the number of training samples increased, the feature set (basis matrix $W$ ) remained static and updated the SVM only. Note that when the number of training samples increased from 497 to 997 , the recognition rate increased clearly, as shown in Figure 9. Hence, the initial static features were obtained at 497, 747, and 997 training samples, respectively, and the SVM was updated to learn all 2747 training samples. Recognition performance of the SVM when the feature set remained static is shown in Table 5. 
Table 5. Recognition performance of the support vector machine (SVM) when the feature set remains static.

\begin{tabular}{cccc}
\hline The Sample Size Used for Static Feature Extraction & $\mathbf{4 9 7}$ & $\mathbf{7 4 7}$ & $\mathbf{9 9 7}$ \\
\hline Initial accuracy & $80.99 \%$ & $85.08 \%$ & $87.79 \%$ \\
Final accuracy of only SVM learning & $86.42 \%$ & $87.39 \%$ & $88.73 \%$ \\
Final accuracy of NMF and SVM learning & & $91.29 \%$ & \\
\hline
\end{tabular}

\section{Discussion}

In this work, we show how incremental learning can be applied to SAR target recognition. High computation efficiencies are demonstrated with the developed $L_{p}$-INMF, with the number of samples continually increasing. The results are compared to NMF, which is a batch-learning method. Moreover, applying sparse constraints to the developed $L_{p}$-INMF leads to an increase in accuracy. The recognition performance of $L_{p}$-INMF also surpassed that of non-incremental NMF and INMF. Since this work aimed to reduce computational complexity with increasing sample number while maintaining the same recognition performance as the corresponding non-incremental methods, we did not compare the method to more time-consuming batch learning methods such as deep learning. A detailed discussion of the corresponding experimental section follows.

\subsection{Efficiency Analysis}

As the number of training samples increases continually, the training time of NMF increases. The training time of $L_{p}$-INMF remains stable, as shown in Figure 5 . When the number of training samples increases, NMF must retrain all existing and new training samples, whereas $L_{p}$-INMF just updates the model by computing the new samples. This demonstrates that the computational complexity of traditional NMF methods increases with the sample size.

Incremental PCA (IPCA) is also tested, but IPCA is not an ideal incremental learning method because of its massive computation requirements. The computation time for updating a single sample for IPCA is about six times longer than that of INMF, $L_{1}-\mathrm{INMF}$, and $L_{1 / 2}$-INMF, as shown in Figure 6 .

We verify that the proposed $L_{p}$-INMF avoids repetitive learning of existing samples and improves data processing efficiency without increasing INMF's computational complexity.

\subsection{Decomposition Value Error Analysis}

Two important phenomena are shown in Figures 7 and 8. Firstly, the VAE and RMSE of $L_{p}$-INMF ( $L_{1}$-INMF and $L_{1 / 2}$-INMF) are both lower than for the traditional INMF at all times. This means that $L_{1}$-INMF and $L_{1 / 2}$-INMF have more accurate decomposition matrices than INMF. Note that RMSE values for INMF and $L_{p}$-INMF ( $L_{1}$-INMF and $L_{1 / 2}$-INMF) are not too different. However, the results of the two indexes should be compared together. It is proved that $L_{1}$-INMF and $L_{1 / 2}$-INMF obtain more precise solutions than INMF. Secondly, the VAE and RMSE values increase as the number of new training samples increases. This phenomenon validates that the error of the two decomposition matrices $W$ and $H$ accumulates as the number of training samples increases. Thus, mapping of training samples is a necessary last step to avoid error accumulation, as discussed in Section 3.2.

\subsection{Recognition Performance Analysis}

In Table 5, keeping the features unchanged, SVM updating can bring the recognition rate up along with the increasing of training sample number. SVM updating is an important factor, in that the recognition accuracy increases along with the increase in sample number. However, the final recognition accuracy value of SVM learning is inferior to the final recognition accuracy of NMF and SVM, which is $91.29 \%$. Not a single case of feature updating or classifier updating can be dispensed with. This article focuses on NMF feature updates rather than SVM updating. Our goal is to prove that our feature update method ( $L_{p}-\mathrm{INMF}$ ) is superior to traditional methods (NMF, INMF). Future 
research will focus on classifier updating. From an experimental completeness perspective, study in this area is necessary.

Figure 9 verifies two points: (1) INMF cannot outperform non-incremental NMF; and (2) the proposed methods ( $L_{1}$-INMF and $L_{1 / 2}$-INMF) obtain higher accuracy than non-incremental methods (NMF) and incremental methods (INMF) when the number of samples increases continuously.

For INMF methods, incremental methods with $L_{p}$ sparse constraint $\left(L_{1}\right.$-INMF and $L_{1 / 2}$-INMF) obtain higher accuracy rates than incremental NMF, as shown in Figure 9 and Table 4.

The main advantage of the proposed method is that $L_{p}$-INMF can improve the accuracy of INMF without increasing computational complexity in SAR target recognition.

\section{Conclusions}

In this paper, $L_{p}$-INMF is proposed to solve efficiency problems when the number of training samples increases continuously in SAR target recognition. There are two improvements in the proposed method: (1) the existing training model can be updated directly, reducing computational cost and improving efficiency when the number of training samples increases; and (2) $L_{p}$ sparse constraints are added to the decomposition matrix during the update process, leading to more accurate solutions compared to general INMF. The experimental results verify that $L_{p}$-INMF reduces the computational cost, while improving the recognition performance.

To demonstrate recognition performance and efficiency of the proposed $L_{p}$-INMF, specific experiments were set. By using MSTAR data, experimental results showed that $L_{p}$-INMF has a faster training model computation speed compared to non-incremental NMF. Moreover, the computation time for updating one sample in $L_{p}$-INMF was six times faster than for IPCA. We also demonstrated that the recognition performance of $L_{p}$-INMF is higher than that of NMF and INMF when $p$ is set to 1 and $\frac{1}{2}$. Finally, error analyses showed that the VAE and RMSE values of $L_{p}$-INMF are both lower than those of traditional INMF. Therefore, the proposed $L_{p}$-INMF updates the existing training model efficiently and precisely as the number of target training samples increases gradually.

This paper verifies that the proposed $L_{p}$-INMF can be an efficient and accurate feature-updating method in SAR target recognition systems. This research provides a foundation for us to study the intelligent SAR target learning system. In the future, we will explore a classification model-updating method for SAR target recognition.

Acknowledgments: This study is supported by the Fundamental Research Funds for the Central Universities A03013023601005, the National Nature Science Foundation of China under Grant U1433113.

Author Contributions: Sihang Dang and Zongyong Cui designed and completed the experiments. Zongjie Cao and Nengyuan Liu contributed in analysing results. All authors contributed in writing the paper.

Conflicts of Interest: The authors declare no conflict of interest.

\section{References}

1. Song, S.; Xu, B.; Yang, J. SAR Target Recognition via Supervised Discriminative Dictionary Learning and Sparse Representation of the SAR-HOG Feature. Remote Sens. 2016, 8, 683. doi:10.3390/rs8080683.

2. Cui, Z.; Cao, Z.; Yang, J.; Fen, J. Target recognition in synthetic aperture radar images via non-negative matrix factorisation. IET Radar Sonar Navig. 2015, 9, 1376-1385. doi:10.1049/iet-rsn.2014.0407.

3. Huang, Y.; Pei, J.; Yang, J.; Wang, B.; Liu, X. Neighborhood Geometric Center Scaling Embedding for SAR ATR. IEEE Trans. Aerosp. Electron. Syst. 2014, 50, 180-192. doi:10.1109/taes.2013.110769.

4. Li, X.; Anthony, D.; Shen, C.; Anton Van Den, H.; Wang, H. Incremental Learning of 3D-DCT Compact Representations for Robust Visual Tracking. IEEE Trans. Pattern Anal. Mach. Intell. 2012, 35, 863-881. doi:10.1109/tpami.2012.166.

5. Joshi, P. Incremental Learning: Areas and Methods-A Survey. Int. J. Data Min. Knowl. Manag. Process 2012, 2, 43-51. doi:10.5121/ijdkp.2012.2504.

6. Pratama, M.; Lu, J.; Lughofer, E.; Zhang, G.; Anavatti, S. Scaffolding type-2 classifier for incremental learning under concept drifts. Neurocomputing 2016, 191, 304-329. doi:10.1016/j.neucom.2016.01.049. 
7. Balsubramani, A.; Dasgupta, S.; Freund, Y. The Fast Convergence of Incremental PCA. In Proceedings of the International Conference on Neural Information Processing Systems, Lake Tahoe, NV, USA, 5-10 December 2013; pp. 3174-3182.

8. Dagher, I. Incremental PCA-LDA algorithm. In Proceedings of the 2010 IEEE International Conference on Computational Intelligence for Measurement Systems and Applications, Taranto, Italy, 6-8 September 2010. doi:10.1109/cimsa.2010.5611752.

9. Choi, Y.; Tokumoto, T.; Lee, M.; Ozawa, S. Incremental two-dimensional two-directional principal component analysis (I(2D)2PCA) for face recognition. In Proceedings of the Acoustics, Speech and Signal Processing, Prague, Czech Republic, 22-27 May 2011; pp. 1493-1496. doi:10.1109/icassp.2011.5946776.

10. Ghassabeh, Y.A.; Rudzicz, F.; Moghaddam, H.A. Fast incremental LDA feature extraction. Pattern Recognit. 2015, 48, 1999-2012. doi:10.1016/j.patcog.2014.12.012.

11. Bucak, S.S.; Bilge, G. Incremental subspace learning via non-negative matrix factorization. Pattern Recognit. 2009, 42, 788-797. doi:10.1016/j.patcog.2008.09.002.

12. Domeniconi, C.; Gunopulos, D. Incremental Support Vector Machine Construction. In Proceedings of the 2001 IEEE International Conference on Data Mining, San Jose, CA, USA, 29 November-2 December 2014; pp. 589-592. doi:10.1109/icdm.2001.989572.

13. Forster, K.; Monteleone, S.; Calatroni, A.; Roggen, D.; Troster, G. Incremental kNN Classifier Exploiting Correct-Error Teacher for Activity Recognition. In Proceedings of the Ninth International Conference on Machine Learning and Applications, Washington, DC, USA, 12-14 December 2010; pp. 445-450. doi:10.1109/icmla.2010.72.

14. Ristin, M.; Guillaumin, M.; Gall, J.; Van Gool, L. Incremental Learning of Random Forests for Large-Scale Image Classification. IEEE Trans. Pattern Anal. Mach. Intell. 2016, 38, 490-503. doi:10.1109/tpami.2015.2459678.

15. Gu, B.; Sheng, V.S.; Tay, K.Y.; Romano, W.; Li, S. Incremental Support Vector Learning for Ordinal Regression. IEEE Trans. Neural Netw. Learn. Syst. 2017, 26, 1403-1416. doi:10.1109/tnnls.2014.2342533.

16. Martinez-Rego, D.; Perez-Sanchez, B.; Fontenla-Romero, O.; Alonso-Betanzos, A. A robust incremental learning method for non-stationary environments. Neurocomputing 2017, 74, 1800-1808. doi:10.1016/j.neucom.2010.06.037.

17. Song, Q.; Xu, F. Zero-Shot Learning of SAR Target Feature Space With Deep Generative Neural Networks. IEEE Geosci. Remote Sens. Lett. 2017, 44, 2245-2249. doi:10.1109/lgrs.2017.275890.

18. Cao, Z.; Feng, J.; Min, R.; Pi, Y. NMF and FLD based feature extraction with application to Synthetic Aperture Radar target recognition. In Proceedings of the IEEE International Conference on Communications, Ottawa, ON, Canada, 10-15 June 2012; pp. 6416-6420. doi:10.1109/icc.2012.6364801.

19. Gao, X.; Cao, Z.; Zheng, Y.; Fan, Y.; Zhang, Q. SAR target feature extraction based on sparse constraint nonnegative matrix factorization. In Proceedings of the IEEE Globecom Workshops, Anaheim, CA, USA, 3-7 December 2012; pp. 1440-1444. doi:10.1109/glocomw.2012.6477796.

20. Lu, X.; Wu, H.; Yuan, Y.; Yan, P.; Li, X. Manifold Regularized Sparse NMF for Hyperspectral Unmixing. IEEE Trans. Geosci. Remote Sens. 2013, 51, 2815-2826. doi:10.1109/tgrs.2012.2213825.

21. Leigsnering, M.; Ahmad, F.; Amin, M.; Zoubir, A. Multipath exploitation in through-the-wall radar imaging using sparse reconstruction. IEEE Trans. Aerosp. Electron. Syst. 2014, 50, 920-2939. doi:10.1109/taes.2013.120528.

22. Dang, S.; Cui, Z.; Cao, Z.; Liu, Y.; Min, R. SAR target recognition via incremental nonnegative matrix factorization with Lp sparse constraint. In Proceedings of the Radar Conference IEEE, Seattle, WA, USA, 8-12 May 2017. doi:10.1109/radar.2017.7944260.

23. Silva, J.; Bacao, F.; Caetano, M. Specific Land Cover Class Mapping by Semi-Supervised Weighted Support Vector Machines. Remote Sens. 2017, 9, 181. doi:10.3390/rs9020181.

24. Wieland, M.; Liu, W.; Yamazaki, F. Learning Change from Synthetic Aperture Radar Images: Performance Evaluation of a Support Vector Machine to Detect Earthquake and Tsunami-Induced Changes. Remote Sens. 2016, 8, 792. doi:10.3390/rs8100792.

25. Keydel, E.R.; Lee, S.W.; Moore, J.T. MSTAR extended operating conditions: A tutorial. Aerosp./Def. Sens. Controls 1996, 2757, 228-242. doi:10.1117/12.242059. 
26. Huan, R.; Pan, Y. Decision fusion strategies for SAR image target recognition. IET Radar Sonar Navig. 2011, 5 , 747-755. doi:10.1049/iet-rsn.2010.0319.

27. Cao, Z.; Xu, L.; Feng, J. Automatic target recognition with joint sparse representation of heterogeneous multi-view SAR images over a locally adaptive dictionary. Signal Process. 2016, 126, 27-34. doi:10.1016/j.sigpro.2015.12.018.

(C) 2018 by the authors. Licensee MDPI, Basel, Switzerland. This article is an open access article distributed under the terms and conditions of the Creative Commons Attribution (CC BY) license (http:// creativecommons.org/licenses/by/4.0/). 